\title{
Embryonic disc formation following post-hatching bovine embryo development in vitro
}

\author{
Priscila Ramos-Ibeas, Ismael Lamas-Toranzo, Álvaro Martínez-Moro, Celia de Frutos, \\ Alejandra C Quiroga, Esther Zurita and Pablo Bermejo-Álvarez \\ Departamento de Reproducción Animal, INIA, Madrid, Spain \\ Correspondence should be addressed to P Ramos-Ibeas or P Bermejo-Álvarez; Email: priscilaramosibeas@gmail.com \\ or borrillobermejo@hotmail.com
}

\begin{abstract}
Failures during conceptus elongation are a major cause of pregnancy losses in ungulates, exerting a relevant economic impact on farming. The developmental events occurring during this period are poorly understood, mainly because this process cannot be recapitulated in vitro. Previous studies have established an in vitro post-hatching development (PHD) system that supports bovine embryo development beyond the blastocyst stage, based on agarose gel tunnels and serum- and glucose-enriched medium. Unfortunately, under this system embryonic disc formation is not achieved and embryos show notorious signs of apoptosis and necrosis. The objective of this study has been to develop an in vitro system able to support embryonic disc formation. We first compared post-hatching development inside agarose tunnels or free-floating over an agarose-coated dish in serum- and glucoseenriched medium (PHD medium). Culture inside agarose tunnels shaped embryo morphology by physical constriction, but it restricted embryo growth and did not provide any significant advantage in terms of development of hypoblast and epiblast lineages. In contrast to PHD medium, a chemically defined and enriched medium (N2B27) supported complete hypoblast migration and epiblast survival in vitro, even in the absence of agarose coating. Cells expressing the pluripotency marker SOX 2 were observed in $\sim 56 \%$ of the embryos and $\sim 25 \%$ developed embryonic disc-like structures formed by SOX2+ cells. In summary, here we provide a culture system that supports trophectoderm proliferation, hypoblast migration and epiblast survival after the blastocyst stage.

Reproduction (2020) 160 579-589
\end{abstract}

\section{Introduction}

Understanding ungulate embryo development during the first 2 weeks after fertilization is critical to improve reproductive efficiency in livestock, since the greatest gestational losses occur within this period, leading to an important economic impact on farming (Ayalon 1978, Diskin \& Morris 2008, Berg et al. 2010). Focussing on cattle, around one-third of the viable blastocysts fail to achieve conceptus elongation in vivo (Santos et al. 2004). Conceptus elongation entails several critical developmental processes, including the development of extraembryonic membranes and embryonic disc (van Leeuwen et al. 2015). By day 9 of development, two different lineages have emerged from the inner cell mass (ICM): the epiblast and the hypoblast (Maddox-Hyttel et al. 2003). The hypoblast will migrate to cover the entire blastocyst, lying beneath both the epiblast and the trophectoderm, whereas the epiblast will form the embryonic disc. Towards the end of the second week of pregnancy, the extraembryonic membranes experience a massive growth by which the embryo elongates, the epiblast forms a one- or two-layered epithelium and the trophectoderm covering the epiblast (Rauber's layer) disappears (van Leeuwen et al. 2015). Unfortunately, bovine in vitro embryo culture endpoint is restricted to hatched blastocysts on day 8 or 9 of development, impairing the investigation of more developmentally advanced stages. Alternatively, day 7 blastocysts can be transferred to surrogate mothers and elongated embryos can be recovered on days 14-16 of development (van Leeuwen et al. 2015), although with an elevated effort and economical cost.

Previous studies have established different in vitro culture systems to support development beyond the blastocyst stage in bovine embryos (Brandao et al. 2004, Vajta et al. 2004, Alexopoulos et al. 2005). The most advanced method is a post-hatching development (PHD) system, where embryos are cultured inside agarose gel tunnels filled with serum- and glucoseenriched medium (PHD medium) until day 15 or 16 . The embryos developed in this system showed a defined mass of epiblast-derived cells, Rauber's layer and some degree of proliferation of hypoblast cells inside the epiblast and trophoblast (Brandao et al. 2004, Vajta et al. 2004, Machado et al. 2013). However, it remains unclear whether the hypoblast covers entirely the inner surface of the embryo, and notorious signs of apoptosis 
and necrosis could be noticed, especially in epiblastderived cells.

PHD system relies on two main differential features compared to conventional pre-hatching in vitro systems: the use of agarose tunnels as a culture substrate and a higher concentration of glucose and serum. Agarose substrate prevents the attachment of the embryo to the bottom of the culture dish, extending the window of embryo development in vitro, but the metabolic pathways by which this medium supports embryo development in vitro remain unexplored. Nevertheless, it remains unclear whether embryo culture inside agarose tunnels enhances embryo development and triggers elongation or if these embryos are only shaped to a tubular form due to the restricted space inside the tunnel. Furthermore, alternative culture substrates and enriched media that could support epiblast survival and the formation of the embryonic disc have not been explored. The objective of this study has been to develop an in vitro system to achieve embryonic disc formation during post-hatching bovine embryo development. To this aim, we have evaluated the effects of different culture substrates (agarose tunnels, agarose layer or no agarose) and media (PHD or N2B27 media) on bovine post-hatching embryo development in vitro.

\section{Materials and methods \\ In vitro production of bovine blastocysts}

The techniques for in vitro embryo production have been described in detail previously (Bermejo-Alvarez et al. 2011, Lamas-Toranzo et al. 2018). Briefly, immature cumulusoocyte complexes (COCs) were obtained by aspirating follicles $(2-8 \mathrm{~mm})$ from bovine ovaries collected at a local slaughterhouse. COCs were matured for $24 \mathrm{~h}$ in TCM-199 supplemented with $10 \%(\mathrm{v} / \mathrm{v})$ fetal calf serum $(\mathrm{FCS})$ and $10 \mathrm{ng} / \mathrm{mL}$ EGF at $39^{\circ} \mathrm{C}$ under an atmosphere of $5 \% \mathrm{CO}_{2}$ in air with maximum humidity. For in vitro fertilization (IVF), matured COCs were inseminated with frozen-thawed BoviPure (Nidacon) separated bull sperm at a final concentration of $10^{6}$ spermatozoa $/ \mathrm{mL}$. Gametes were co-incubated in fourwell dishes containing $50 \mathrm{COCs}$ and $500 \mu \mathrm{L}$ of TALP medium per well at $39^{\circ} \mathrm{C}$ in an atmosphere of $5 \% \mathrm{CO}_{2}$ and maximum humidity. Semen from the same bull was used for all the experiments to avoid a possible confounding bull effect on developmental rates and to reduce inter-replicate variation. At approximately $20 \mathrm{~h}$ post-insemination (hpi), presumptive zygotes were denuded and cultured in groups of $\sim 25$ in 25 $\mu \mathrm{L}$ droplets under mineral oil. Culture took place in synthetic oviduct fluid (SOF) (Holm et al. 1999) supplemented with $5 \%$ FCS at $39^{\circ} \mathrm{C}$ under an atmosphere of $5 \% \mathrm{CO}_{2}, 5 \% \mathrm{O}_{2}$, and $90 \% \mathrm{~N}_{2}$ with maximum humidity.

In vivo embryos were obtained from two Holstein cows following the superovulation protocol described in Supplementary Fig. 1 (see section on supplementary materials given at the end of this article), which involved progesterone $(C I D R \AA$, Zoetis), PGF2 $\alpha$ analog (cloprostenol,
Estrumate ${ }, M S D)$, GnRH (Dalmarelin $®$, Fatro lbérica), and follicle-stimulating hormone and luteinizing hormone

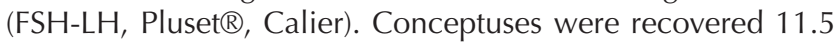
days (E11) or 14.5 days (E14) after first insemination by uterine flushing using an $18 \mathrm{~mm}$ embryo flushing catheter (Minitüb).

\section{Post-hatching development system}

Three independent experiments were carried out to compare different conditions for post-hatching embryo development: (1) agarose tunnels vs agarose layer in PHD medium, (2) PHD medium vs N2B27 medium over agarose layer, and (3) agarose layer vs no layer in N2B27 medium (Fig. 1). Agarose gels were prepared 3 days before use and covered with PHD or N2B27 media (Brandao et al. 2004). Four-well dishes were covered with agarose gel for culture on a layer. To prepare agarose tunnels, $1 \mathrm{~mm}$ wide glass capillaries (World Precision Instruments) were cut to $60 \mathrm{~mm}$ length and one of the open ends was closed by melting the glass over a gas flame. Eight capillaries were oriented in parallel and fixed with tape at the open end. Two of the resulting combs were placed in the opposite direction in a $60 \mathrm{~mm}$ Petri dish with the closed ends placed in the bottom (Supplementary Fig. 2A). Gel was prepared by solving $2.4 \%$ ultrapure low melting point agarose (Thermo Fisher Scientific) in PBS. The solution was autoclaved, $10 \%$ FBS was added once cooled down to $45^{\circ} \mathrm{C}$, and then immediately poured into the culture dishes. Dishes were placed in ice bags for rapid solidification of the gel, PHD medium was poured on the gel surface and capillaries were carefully removed. Tunnels were cut to $15 \mathrm{~mm}$ length and excess gel was removed. Dishes were kept at $39^{\circ} \mathrm{C}$ and $5 \%$ $\mathrm{CO}_{2}$ in water-saturated atmosphere, medium was replaced and the lumen of the tunnels was washed daily with a pulled and curved Pasteur pipette until utilization.

All post-hatching development systems tested took place at $39^{\circ} \mathrm{C}$ in a water-saturated atmosphere of $5 \% \mathrm{CO}_{2}, 5 \% \mathrm{O}_{2}$, and $90 \% \mathrm{~N}_{2}$. Day 9 (D9) in vitro produced blastocysts were transferred to 1:1 SOF:PHD medium (SOF supplemented with $0.5 \%$ glucose and $10 \%$ FBS) for PHD groups or to N2B27 medium (1:1 Neurobasal and DMEM/F12 medium supplemented with penicillin/streptomycin, $2 \mathrm{mM}$ glutamine, N2 and B27, Thermo Fisher Scientific) for N2B27 groups. Day 9 to day 11 (D11) culture took place without agarose. On D11, hatched blastocysts showing trophoblast expansion and a defined inner cell mass were transferred to agarose tunnel or layer in PHD medium (experiment 1), to agarose layer in PHD or N2B27 media (experiment 2) or to N2B27 medium with or without agarose layer (experiment 3) (Fig. 1). Pictures were taken to determine the initial embryo size at D11. Embryos remained in culture until day 15 (D15), when pictures were taken to measure embryo length, area and volume. At the end of the culture, those embryos reaching a significant growth were collected for further analyses.

\section{Embryo measurements}

Pictures from the embryos were taken at D11 and D15 on a stereomicroscope (Zeiss Stemi 305). Length and area were measured using Fiji software (Schindelin et al. 2012). 
Experiment 1: Agarose tunnel vs. layer

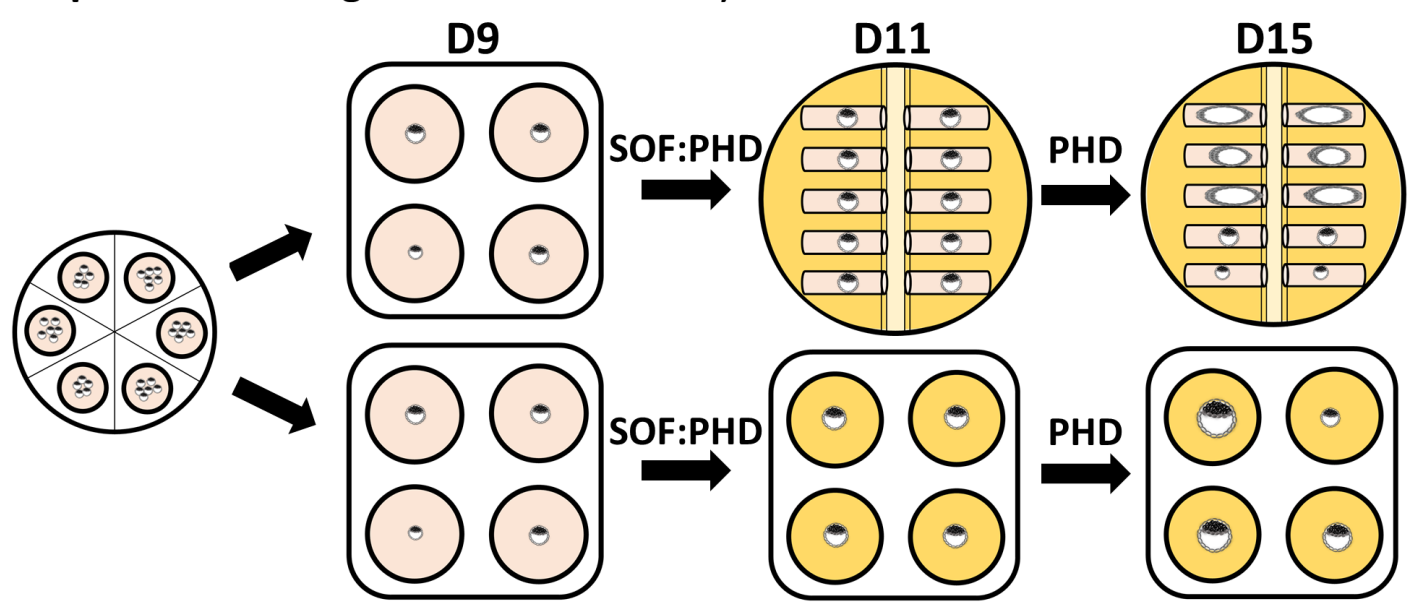

Experiment 2: PHD vs. N2B27 on agarose layer

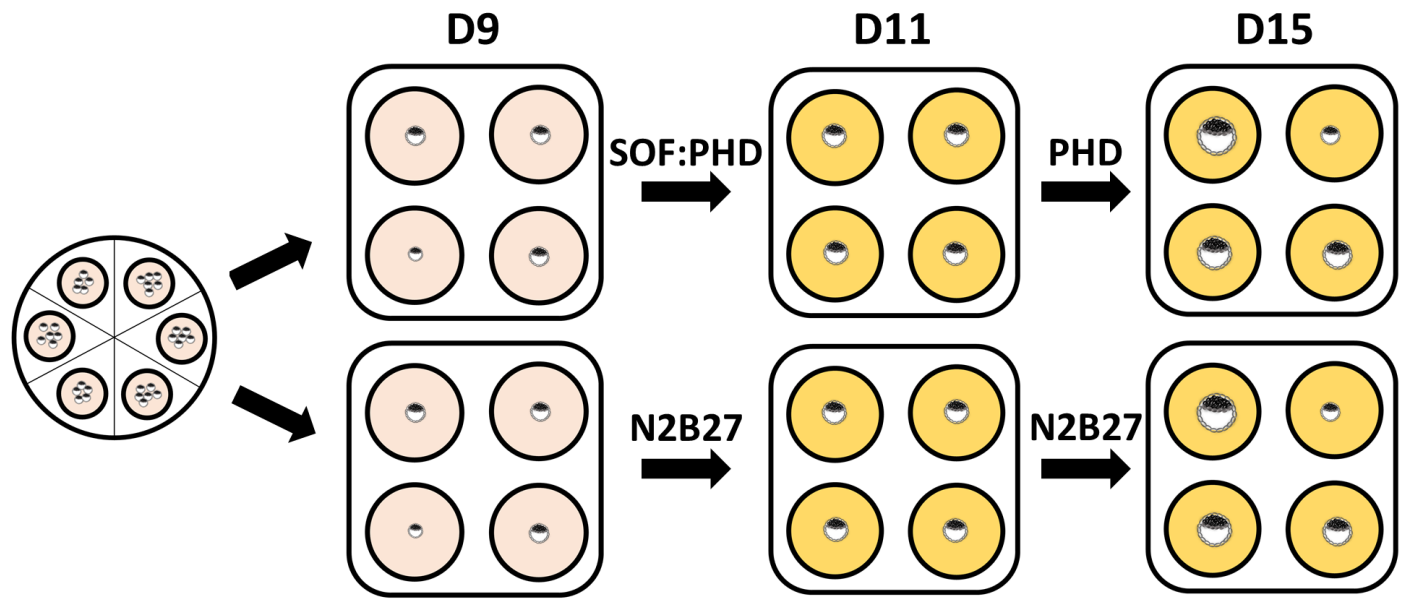

Experiment 3: Agarose layer vs. no agarose in N2B27

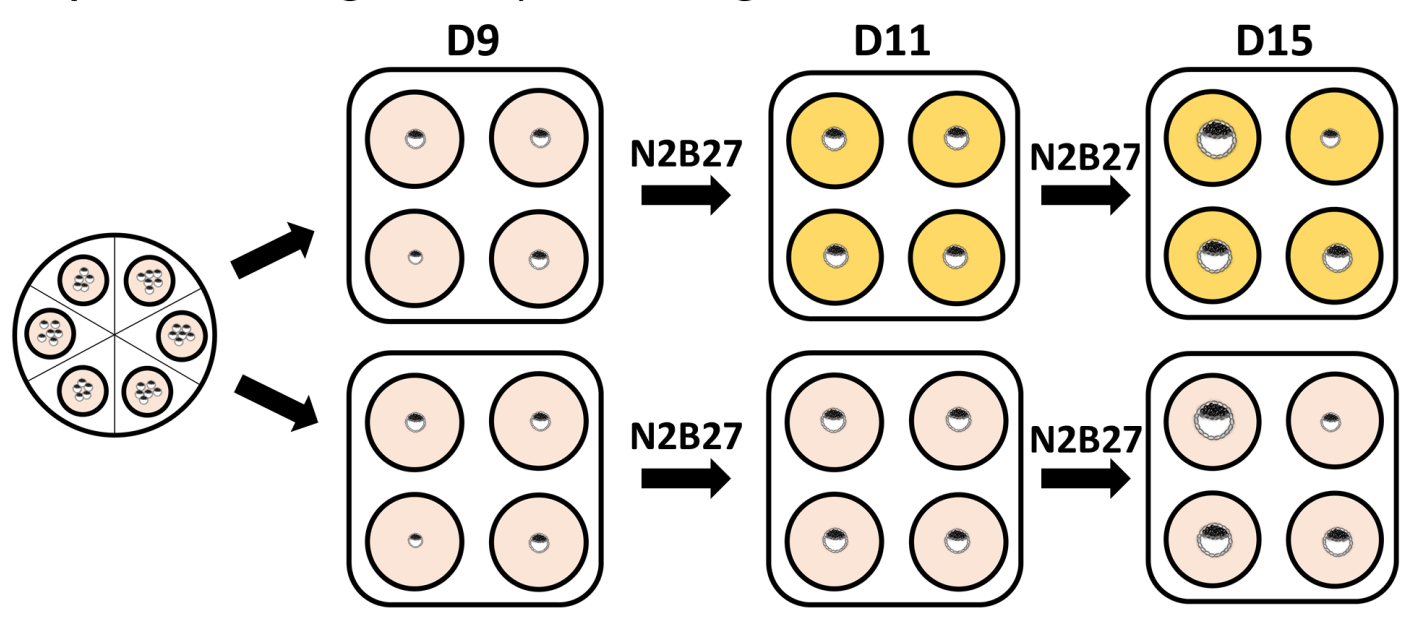

Figure 1 Experimental design. All experiment started from D9 blastocysts produced in vitro in SOF medium. Experiment 1 compared the original PHD system in agarose tunnel (original system, depicted as a yellow dish with tunnels) vs culture on agarose layer (represented as yellow wells) using the same media: SOF:PHD from D9 to D11 (without agarose) and PHD from D11 to D15 (with agarose tunnel or layer). Experiment 2 compared PHD vs N2B27 media using agarose layer as culture support from D11 to D15. Experiment 3 compared agarose layer vs no layer (illustrated as pink wells) using N2B27 medium. 
Elongated embryos were classified as spherical or cylindrical. Volume was calculated using the formula $V=\frac{4}{3} \pi r^{3}$ for spherical embryos. Cylindrical embryos were subdivided into a sphere and a cylinder. Total embryo volume was calculated by adding the volume of a sphere and the volume of a cylinder, calculated with the formula $V=\pi r^{2} h$. As very few embryos smaller than $0.5 \mathrm{~mm}$ in diameter at D11 survived to D15, and D11 initial embryo size could influence D15 embryo dimensions, only D15 embryos developing from D11 embryos larger than $0.5 \mathrm{~mm}$ were used for analysis.

\section{Immunofluorescence}

Embryos were fixed in 4\% paraformaldehyde (PFA) for $15 \mathrm{~min}$ at room temperature (RT), washed in PBS-1\% BSA, permeabilized in 1\% Triton X-100 in PBS for $15 \mathrm{~min}$ at RT and blocked in 10\% Donkey Serum-0.02\% Tween 20 in PBS for $1 \mathrm{~h}$ at RT. Then, embryos were incubated overnight at $4^{\circ} \mathrm{C}$ with primary antibodies to detect trophectoderm (CDX2, Biogenex MU392A-UC 1:100 dilution), hypoblast (SOX17, R\&D AF1924, 1:100 dilution), or epiblast (SOX2, Invitrogen 14-9811-80, 1:100 dilution) cells. After four washes in PBS-1\% BSA, embryos were incubated in the appropriate secondary Alexa-conjugated antibodies (Life Technologies) and DAPI for $1 \mathrm{~h}$ at RT, followed by four washes in PBS-1\% BSA. Finally, embryos were mounted and imaged at a Nikon Eclipse TE 300 fluorescence microscope or at a Leica TCS-SP5 AOBS laser scanning confocal microscope (Leica Microsystems). For tridimensional images, embryos were placed on PBS-1\% BSA microdrops made by drawing circles with a PAP pen (Kisker Biotech $\mathrm{GmbH}$ ) on a coverslide, as previously described (Bermejo-Alvarez et al. 2012). Microdrops were covered by an incubation chamber (Sigma Z37,9467) to prevent embryo crushing (Supplementary Fig. 2B). The number of embryos analyzed were 25 in experiment 1 (12 and 13 in tunnel and layer groups, respectively), 25 in experiment 2 (15 and 10 in PHD and N2B27 groups, respectively) and 27 in experiment 3 (11 and 16 in layer and no layer groups, respectively).

\section{RNA isolation, CDNA synthesis and qPCR}

Poly (A) RNA was extracted from five individual whole D15 embryos of each group and five pools of five D9 blastocysts using the Dynabeads mRNA Purification Kit (Life Technologies) following the manufacturer's instructions with minor modifications (Bermejo-Alvarez et al. 2011). Briefly, $100 \mu \mathrm{L}$ of lysis buffer were added to the sample and incubated at RT for $10 \mathrm{~min}$ with gently shaking. Then, $20 \mu \mathrm{L}$ of beads were added and samples were incubated at RT for 5 min with gentle shaking, allowing beads/mRNA complexes formation. Finally, beads/mRNA complexes were washed twice in washing buffer $A$ and twice in washing buffer $B$ and resuspended in $10 \mathrm{mM}$ Tris- $\mathrm{HCl} \mathrm{pH}$ 7.5. The amount of $\mathrm{mRNA}$ /sample was roughly similar, being around $4 \mathrm{ng}$. Immediately after extraction, samples were treated with DNAse (Promega) at $37^{\circ} \mathrm{C}$ for $5 \mathrm{~min}$ followed by enzyme denaturalization at $90^{\circ} \mathrm{C}$ for $5 \mathrm{~min}$, and then the RT reaction was carried out with qScript CDNA Supermix (Quantabiosciences, Gaithersburg, MS, USA) in a total volume of $20 \mu \mathrm{L}$. Tubes were first incubated at $25^{\circ} \mathrm{C}$ for $5 \mathrm{~min}$ and then at $42^{\circ} \mathrm{C}$ for $60 \mathrm{~min}$ to allow the RT of RNA, followed by $85^{\circ} \mathrm{C}$ for $5 \mathrm{~min}$ to denature the reverse transcriptase. mRNA transcripts were quantified by real-time quantitative PCR (qPCR). Two replicate PCR experiments were conducted for all genes of interest and qPCR efficiency was tested beforehand; all primers used showed efficiencies above 0.9. PCR was performed by adding a $2-\mu \mathrm{L}$ aliquot of each sample to the PCR mix (GoTaq qPCR Master Mix, Promega) containing the specific primers. Primer sequences are provided in Supplementary Table 1. The comparative cycle threshold (CT) method was used to quantify expression levels. Fluorescence was acquired in each cycle to determine the threshold cycle. According to the comparative CT method, the $C T$ value was determined by subtracting the endogenous control H2AFZ CT value - tested for stability on previous publications (Bermejo-Alvarez et al. 2010b, 2011) - for each sample from the CT value of each gene in the sample. CT was calculated using the highest sample CT value (i.e. the sample with the lowest target expression) as an arbitrary constant to be subtracted from all other CT sample values. Fold changes in the relative gene expression of the target were determined using the formula $2^{-\Delta \Delta C T}$ (Schmittgen \& Livak 2008).

\section{Statistical analysis}

Data were analyzed using the GraphPad Prism (GraphPad Software) software package and a value of $P<0.05$ was considered significant. Chi-square test was used to analyze the differences in survival between groups. Differences in length, area and volume between groups were analyzed by Student's t-test when data distribution was normal. When normality test failed, statistical differences were analyzed by Mann-Whitney Rank Sum Test. Embryo size at D11 was not used as a co-factor given that D11 embryos smaller than $0.5 \mathrm{~mm}$ were excluded for D15 measurement analysis, as most did not survive to that day. Differences in mRNA expression were analyzed by oneway ANOVA (experiment 1) or $t$-test (experiment 2).

\section{Results}

\section{Post-hatching development in agarose tunnel or without physical constriction}

In order to assess the effect of embryo culture inside agarose tunnels on post-hatching development, in vitro produced bovine embryos were cultured in agarose tunnels or free-floating over a layer of agarose, in high serum and glucose medium (PHD medium) according to previously described methods (Brandao et al. 2004, Vajta et al. 2004). On day 11 of development, embryos were measured and randomly allocated to the two different culture systems (tunnel or layer) until day 15, when embryo survival and growth were assessed. The main factor determining embryo survival was the initial embryo size at D11. When initial embryo diameter was smaller than $0.5 \mathrm{~mm}$, only 1 out of $37(\sim 3 \%)$ and 1 out of $22(\sim 5 \%)$ embryos survived in agarose tunnel and layer, respectively. However, when embryo diameter was $0.5 \mathrm{~mm}$ or more, 23 out of 32 ( 72\%) embryos 
cultured in agarose tunnel and 19 out of $26(\sim 73 \%)$ embryos cultured over agarose layer survived. No differences were observed in embryo survival between agarose tunnel and layer systems. Although surviving embryos cultured inside tunnels displayed an elongated form distinct to the spherical shape of those cultured over an agarose layer (Fig. 2A), embryo length (i.e. the maximum dimension) was similar between both groups. However, area and volume of surviving embryos were significantly higher in embryos cultured over an agarose layer compared to those cultured inside an agarose tunnel, indicating that culture inside a tunnel restricted embryo growth (Table 1).

To assess lineages differentiation, hypoblast cells were detected by immunostaining for SOX17 (NegrónPérez et al. 2017). A layer of SOX17+ cells was detected inside the CDX2+ trophectoderm in most of the embryos that grew until D15 in both culture conditions. However, in contrast to in vivo derived embryos, hypoblast cells did not cover the entire inner embryo surface (Fig. 2B and Supplementary Fig. 3). The surface of the embryo covered by hypoblast was very variable and there were no significant differences between both conditions (Table 2). Day 9 in vitro-produced embryos, that is, prior to culture in PHD medium, showed clear SOX2+ cells in the ICM. However, although a compact mass of epiblast-derived cells was detected in some developmentally advanced embryos following culture in PHD medium, no SOX2-positive cells were detected in any culture condition $(n=12$ cultured in tunnel and 13 embryos cultured over agarose layer) and the nuclei of the degenerating masses of epiblast-derived cells appeared disorganized, in contrast to epiblast cells from an in vivo derived embryonic disc (Fig. 3A).

\section{Culture in PHD medium triggers interferon Tau production and induces a metabolic switch in the embryo}

Transcriptional analysis of IFNT2 expression, the principal embryonic signal for pregnancy recognition in ruminants (Imakawa et al. 1987, Helmer et al. 1989), revealed an upregulation in D15 embryos cultured in PHD medium compared to D9 blastocysts irrespective of the culture condition (tunnel or layer), reflecting the proliferation and functional development of the trophectoderm lineage. In order to elucidate a possible metabolic switch during post-hatching embryo culture, the expression of several rate-limiting enzymes involved in anaerobic glycolysis (GAPDH and $L D H A$ (Granchi et al. 2010)), pentose phosphate pathway (G6PD and its positive regulator SIRT2 (Wang et al. 2014)) and Kreb's cycle (citrate synthase, CS (Seedorf et al. 1986)) were analyzed on D15 and D9 embryos. Irrespective of the culture substrate (i.e. tunnel or layer), the anaerobic glycolysis enzymes GAPDH and $\angle D H A$ were upregulated in D15 embryos compared to D9 embryos. However, the rate-limiting enzyme for pentose phosphate pathway G6PD and its positive regulator SIRT2 were downregulated in D15 embryos cultured in agarose layer compared to those cultured in tunnel and D9 embryos. Citrate synthase (CS) expression was also reduced in D15 embryos cultured in agarose layer, pointing to a decrease in oxidative phosphorylation (Fig. 3B).

\section{Post-hatching development in a chemically defined enriched medium}

Irrespective of the culture substrate used, hypoblast and epiblast development was compromised in PHD medium: hypoblast migration along the entire inner embryo surface was not accomplished and epiblast development was abolished. These findings suggest that, while PHD medium supports the proliferation of trophectoderm cells, it is unable to sustain embryo development beyond the blastocyst stage. Aiming to provide a better-suited culture medium, we compared post-hatching embryo development in PHD medium vs N2B27, a defined serum-free enriched medium (Supplementary Table 2) suitable for stem cell culture (Ying \& Smith 2003). Given that physical constriction was previously proven to be dispensable and even slightly detrimental for embryo development, embryos were cultured on agarose layer in both media. D9 in vitro produced blastocysts were randomly allocated to 1:1 PHD:SOF or to N2B27 medium and 2 days later (D11), embryos were measured and transferred to agarosecoated dishes with PHD or N2B27 medium, respectively. Embryo survival from D11 to D15 was similar in both media. As previously observed, D11 embryo size was the main determinant of embryo survival. Two out of $27(\sim 7 \%)$ and 7 out of $24(\sim 29 \%)$ D11 blastocysts with initial diameter smaller than $0.5 \mathrm{~mm}$ survived to D15 in PHD and N2B27 media, respectively. In contrast, 23 out of $35(\sim 66 \%)$ and 15 out of $23(\sim 65 \%)$ D11 embryos larger than $0.5 \mathrm{~mm}$ survived in PHD and N2B27 media, respectively. Surviving embryos developed in a spherical shape in both culture media (Fig. 4A), but diameter, area and volume were significantly higher for those embryos cultured in PHD medium (Table 3). As previously noticed (Table 2), hypoblast proliferation was limited in PHD medium: only 1 out of 15 embryos analyzed showed complete hypoblast migration (Fig. 4B). In contrast, all embryos cultured in N2B27 showed complete migration of the hypoblast along the inner embryo surface $(n=10$, Fig. 4C). Furthermore, while SOX2+ epiblast cells were not observed in any embryo cultured in PHD medium, six out of ten $(60 \%)$ of the embryos cultured in N2B27 medium showed SOX2+ cells and two of them (20\%) showed a compact structure resembling an embryonic disc (Fig. 4C).

Transcriptional analysis showed no differences in the expression of enzymes involved in anaerobic glycolysis 

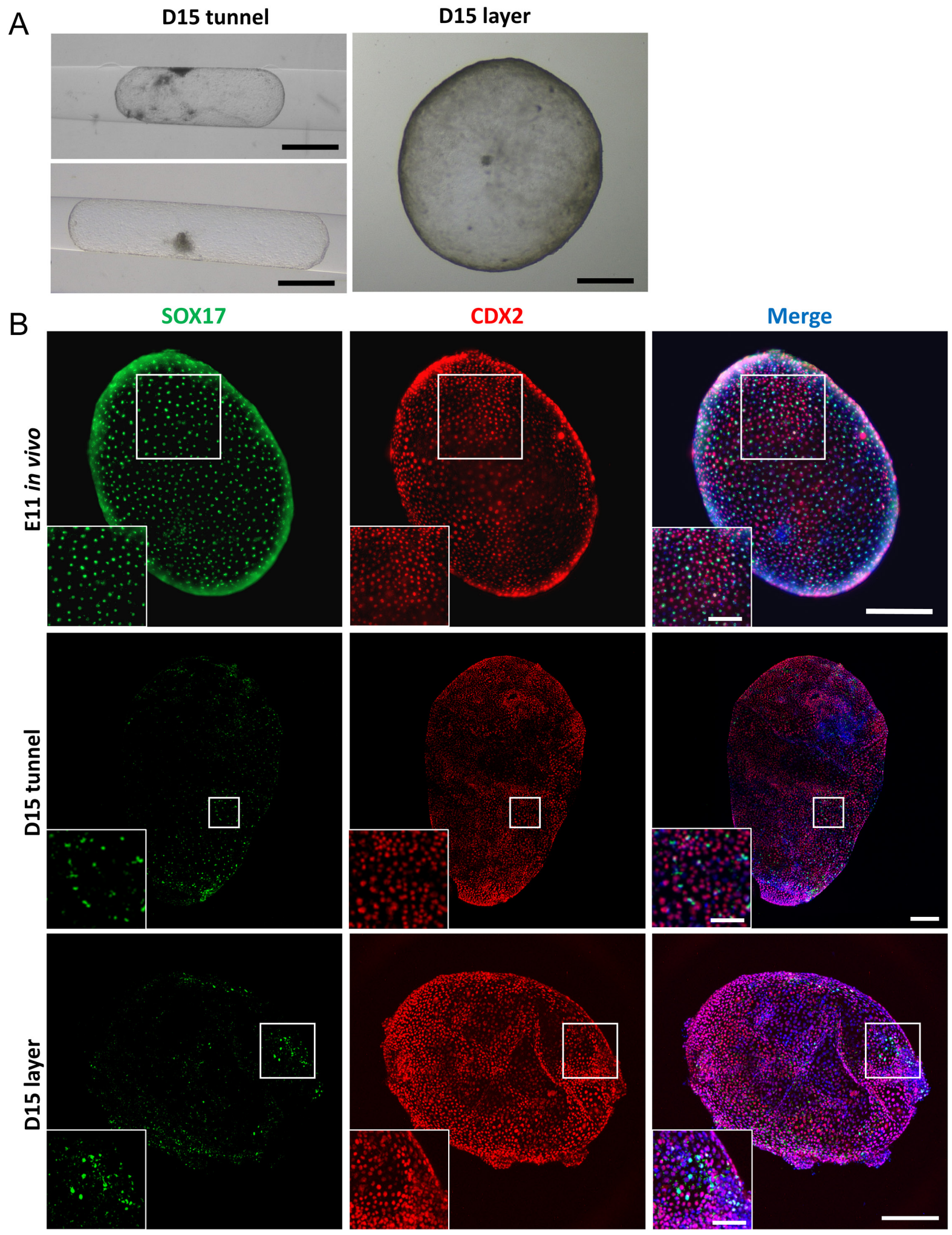

Figure 2 Trophoblast and hypoblast development in agarose tunnel or layer in PHD medium. (A) Representative brightfield stereomicroscopic images of bovine D15 embryos cultured in PHD medium in agarose tunnel or layer. (B) The complete hypoblast migration observed already in embryonic day 11 (E11) in vivo embryos (upper row) contrasts to the incomplete hypoblast layer migration observed in D15 in vitro embryos cultured in PHD medium in agarose tunnel or layer (lower rows). Immunofluorescence staining for SOX17 (hypoblast) and CDX2 (trophoblast); nuclei were counterstained with DAPI (merge). Pictures in the corner are magnifications. Scale bars $=1 \mathrm{~mm}$ for A; $300 \mu \mathrm{m}$ for whole embryo pictures in B; $100 \mu \mathrm{m}$ for magnifications. 
Table 1 Average length, area and volume of surviving embryos at days 11 and 15 of development.

\begin{tabular}{lccccccc}
\hline & $\boldsymbol{n}$ & D11 length $(\mathrm{mm})$ & D11 area $\left(\mathrm{mm}^{2}\right)$ & D11 volume $\left(\mathrm{mm}^{3}\right)$ & D15 length $(\mathrm{mm})$ & D15 area $\left(\mathrm{mm}^{2}\right)$ & D15 volume $\left(\mathrm{mm}^{3}\right)$ \\
\hline Tunnel & 23 & $0.67 \pm 0.02$ & $0.35 \pm 0.02$ & $0.17 \pm 0.02$ & $2.20 \pm 0.31$ & $1.92 \pm 0.31^{\mathrm{a}}$ & $1.52 \pm 0.24^{\mathrm{a}}$ \\
Layer & 19 & $0.77 \pm 0.04$ & $0.46 \pm 0.04$ & $0.27 \pm 0.04$ & $2.09 \pm 0.22$ & $3.98 \pm 0.92^{\mathrm{b}}$ & $7.50 \pm 2.33^{\mathrm{b}}$ \\
\hline
\end{tabular}

Data are mean \pm S.E.M. Within columns, values followed by different superscript letters differ significantly $(P<0.05$, Mann-Whitney Rank Sum Test).

(GAPDH and $L D H A$ ), pentose phosphate pathway (G6PD and SIRT2) and Kreb's cycle (CS) between D15 embryos cultured in PHD vs N2B27 media. IFNT2 transcript was present in both conditions, while it was more abundant in embryos cultured in PHD medium, suggesting that it may induce trophectoderm proliferation to a greater extent than N2B27 (Fig. 4D).

\section{Post-hatching development without agarose substrate}

Finally, we sought to determine if agarose substrate was necessary for bovine embryo development after blastocyst hatching. With this aim, D9 embryos were cultured in N2B27 medium until D11, measured and randomly allocated to agarose-coated or agarose-free wells with N2B27 medium. Survival rates for D11 embryos were mostly determined by embryo diameter, being similar in both groups. Only 2 out of $15(\sim 14 \%)$ and 5 out of $26(\sim 19 \%)$ D11 embryos smaller than 0.5 $\mathrm{mm}$ survived in agarose-coated and agarose-free wells, respectively. However, 11 out of $17(\sim 65 \%)$ and 16 out of 21 ( 76\%) D11 embryos larger than $0.5 \mathrm{~mm}$ survived in agarose-coated and agarose-free wells. D15 embryos showed a spherical shape and no significant differences were found in embryo survival, length, area or volume between both conditions (Fig. 5A and Table 4). All surviving embryos showed complete migration of the hypoblast in both conditions $(n=11$ in agarose-coated and 16 in agarose-free wells, Fig. 5B). No differences were found in the number of embryos showing SOX2+ epiblast cells ( 6 out of $11(\sim 55 \%)$ in agarose-coated and 9 out of $16(\sim 56 \%)$ in agarose-free wells) or showing a SOX2+ compact embryonic disc-like structure (2 out of $11(18 \%)$ in agarose-coated and 4 out of $16(25 \%)$ in agarose-free wells). Therefore, agarose substrate is not required for bovine post-hatching development in vitro.

\section{Discussion}

The development of an in vitro culture system to attain bovine embryo development beyond the blastocyst stage is critical to study conceptus elongation without the need of experimental animals. Unfortunately, current methods achieve limited success, being the posthatching development (PHD) system, described ago, the most successful so far (Brandao et al. 2004, Vajta et al. 2004). Molecular characterization of the development of embryonic lineages under the PHD system revealed that, while trophectoderm proliferated robustly, hypoblast migration was compromised and epiblast development was abolished. An initial step for system optimization was to determine the optimal culture substrate, as PHD system employed agarose tunnels. The potentially beneficial effect of agarose tunnels could be mediated by the physical constriction or by the agarose itself. Our results show that neither agarose nor physical constriction are required for embryo development, as survival rates were similar for embryos cultured inside agarose tunnels, over agarose layer or without agarose coating. Besides, although culture in PHD medium inside agarose tunnels shaped the embryo in to a cylindrical shape, embryonic growth was restricted, as embryo area and volume were significantly larger in embryos developed free-floating over an agarose layer compared to those physically restricted by agarose tunnels.

Although PHD medium did not support epiblast development and compromised hypoblast migration, trophoblast cells proliferated rapidly. Trophoblast cells developed in both PHD and N2B27 media were seemingly functional, as they expressed the trophectoderm marker CDX2 (Berg et al. 2011) and IFNT2, the major pregnancy recognition signal in ruminants (Imakawa et al. 1987, Helmer et al. 1989). Given that trophoblast growth is mainly responsible for embryonic growth, the larger embryonic size and higher transcript abundance of IFNT2 in embryos cultured in PHD medium vs N2B27 medium suggest that PHD medium may promote trophoblast proliferation at the expense of hypoblast and epiblast proliferation. Trophectoderm culture requirements seem to be relatively less restrictive than those of other lineages, as primary bovine trophectoderm cell cultures have been established using other relatively simple media also supplemented with 10\% serum (Talbot et al. 2000, Ramos-lbeas et al. 2014). This situation contrasts with the higher demands of epiblast cells, which have been

Table 2 Hypoblast development in embryos cultured in agarose tunnel or layer at day 15.

\begin{tabular}{lcccccc}
\hline & & & \multicolumn{4}{c}{ Embryo surface covered by hypoblast cells } \\
\cline { 4 - 6 } & $\boldsymbol{n}$ & Embryos with hypoblast cells & $\leq 24 \%$ & $25-49 \%$ & $50-74 \%$ & $\geq 75 \%$ \\
\hline Tunnel & 12 & $9(75 \%)$ & $4(33.3 \%)$ & $2(16.7 \%)$ & $2(16.7 \%)$ & $1(8.3 \%)$ \\
Layer & 13 & $10(76.9 \%)$ & $3(23.1 \%)$ & $2(15.4 \%)$ & $2(15.4 \%)$ & $3(23.1 \%)$ \\
\hline
\end{tabular}

No significant differences were found $(P>0.05$, Fisher exact test). 

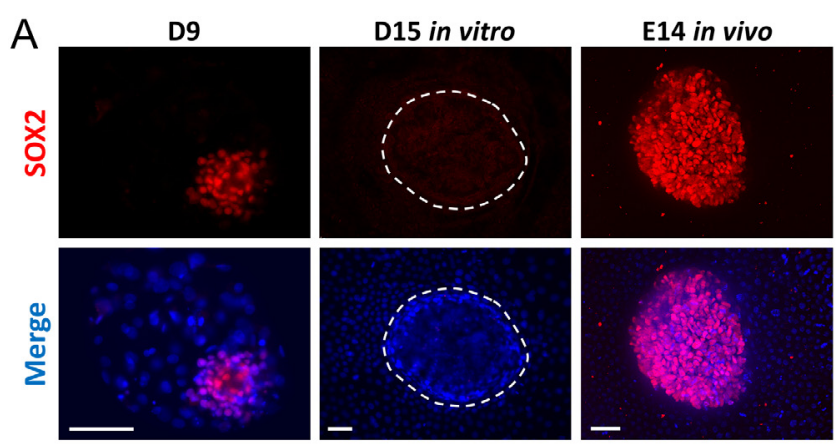

B

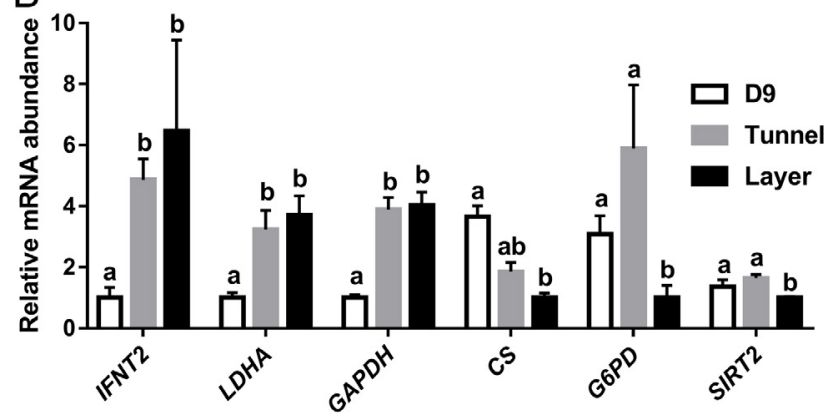

Figure 3 Immunofluorescence detection of epiblast cells and relative mRNA abundance following culture in agarose tunnel or layer in PHD medium. (A) Epiblast development is impaired in D15 embryos developed in PHD medium. Immunofluorescence staining for SOX2 (epiblast) in representative D9 blastocyst (left column), D15 embryo developed in vitro in PHD medium (medium column) and E14 in vivo developed embryonic disc. Nuclei were counterstained with DAPI (merge). Scale bars $=50 \mu \mathrm{m}$. (B) Relative mRNA abundance in D9 and D15 embryos cultured in agarose tunnel or layer in PHD medium. Different letters indicate significant differences based on one-way ANOVA $(P<0.05)$.

proven difficult to capture in vitro in farm animals (Ramos-lbeas et al. 2018). Irrespective of which medium and substrate was used, in vitro post-hatching embryo development was largely determined by initial blastocyst size: most embryos smaller than $0.5 \mathrm{~mm}$ diameter on day 11 were unable to survive up to day 15 in vitro. This finding agrees with previous observations on PHD system (Brandao et al. 2004) and highlights the importance of pre-hatching embryo development for subsequent conceptus elongation and pregnancy success (O'Hara et al. 2014).

In contrast to PHD medium, N2B27 allows both complete hypoblast migration and epiblast development. Hypoblast proliferation, previously reported in the PHD system based on conventional histology and electron microscopy (Brandao et al. 2004), was confirmed by immunostaining for SOX17, an hypoblast marker (Canizo et al. 2019). However, irrespective of the substrate used, hypoblast cells did not cover the entire surface of the embryos developed in PHD medium,
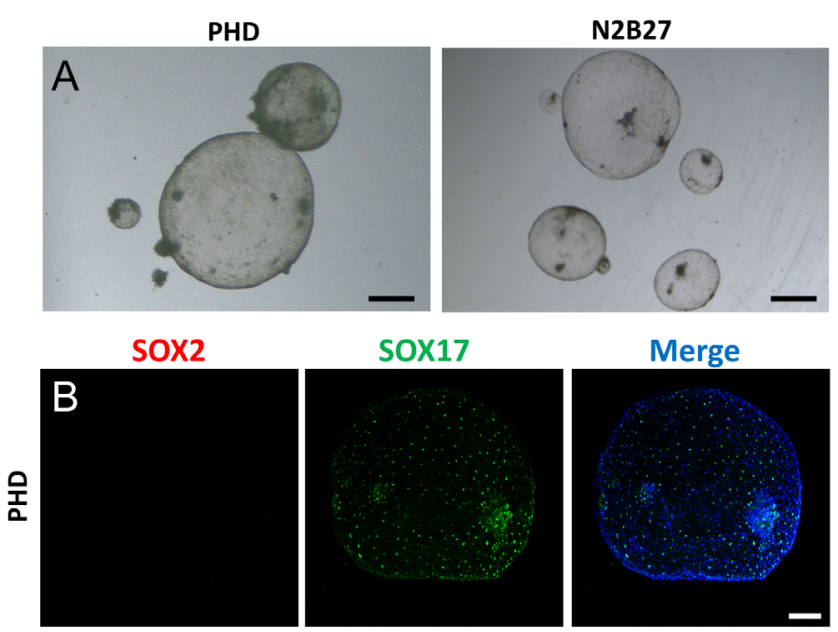

Sox17
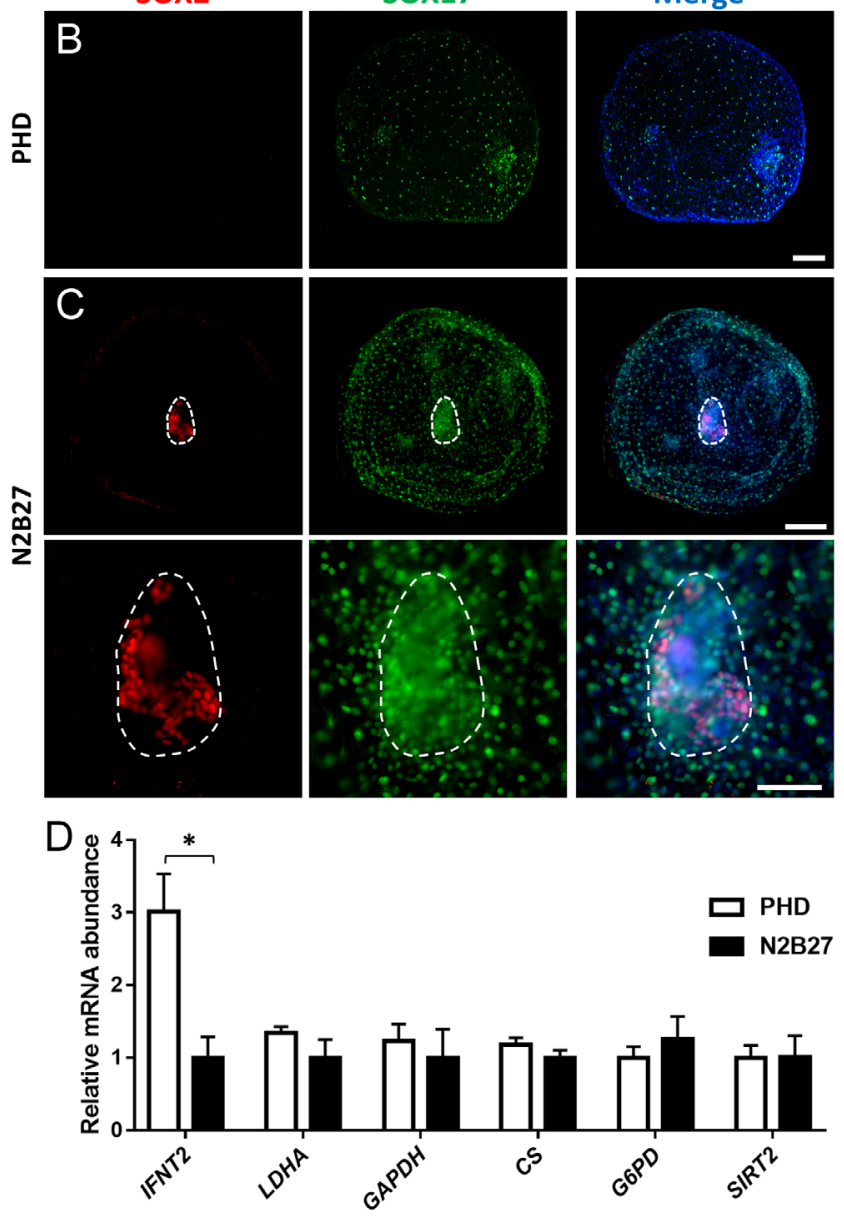

Figure 4 Hypoblast and epiblast development and relative mRNA abundance in embryos developed in PHD or N2B27 media. (A) Representative brightfield stereomicroscopic images of D15 embryos cultured in PHD or N2B27 media over agarose layer. (B) Epiblast and hypoblast development on the only D15 embryo developed in PHD system showing complete hypoblast migration following culture.

Notice the reduced density of hypoblast cells compared with C. (C) Epiblast and hypoblast development on a D15 embryo developed in N2B27 medium over agarose layer. Lower row is a magnification of the compact SOX2+ structure resembling an embryonic disc. Immunofluorescence staining for SOX2 (epiblast) and SOX17 (hypoblast); nuclei were counterstained with DAPI (merge). Scale bars $=1 \mathrm{~mm}$ for $\mathrm{A} ; 200 \mu \mathrm{m}$ for $\mathrm{B}$ and $\mathrm{C}$ upper row; $100 \mu \mathrm{m}$ for magnification in $C$ (lower row). (D) Relative mRNA abundance in D15 embryos cultured in PHD or N2B27 media. Asterisk indicates significant differences based on $t$-test $(P<0.05)$. 
Table 3 Average length, area and volume of surviving embryos cultured on agarose layer in PHD vs N2B27 media at days 11 and 15 of development.

\begin{tabular}{lccccccc}
\hline & $\boldsymbol{n}$ & D11 length $(\mathrm{mm})$ & D11 area $\left(\mathrm{mm}^{2}\right)$ & D11 volume $\left(\mathrm{mm}^{3}\right)$ & D15 length $(\mathrm{mm})$ & D15 area $\left(\mathrm{mm}^{2}\right)$ & D15 volume $\left(\mathrm{mm}^{3}\right)$ \\
\hline PHD & 23 & $0.76 \pm 0.07$ & $0.44 \pm 0.05$ & $0.25 \pm 0.04$ & $2.23 \pm 0.16^{\mathrm{a}}$ & $4.55 \pm 1.07^{\mathrm{a}}$ & $8.84 \pm 2.74^{\mathrm{a}}$ \\
N2B27 & 15 & $0.65 \pm 0.06$ & $0.35 \pm 0.03$ & $0.15 \pm 0.03$ & $1.6 \pm 0.08^{\mathrm{b}}$ & $1.22 \pm 0.47^{\mathrm{b}}$ & $1.26 \pm 0.17^{\mathrm{b}}$ \\
\hline
\end{tabular}

Data are mean \pm S.E.M. Within columns, values followed by different superscript letters differ significantly $(P<0.05$, Mann-Whitney Rank Sum Test).

while all embryos developed in N2B27 medium showed complete hypoblast migration. Similarly, although we observed a compact mass of epiblast-derived cells in D15 PHD embryos, as previously reported (Brandao et al. 2004, Vajta et al. 2004), these cells did not express the epiblast marker SOX2 (Khan et al. 2012). The lack
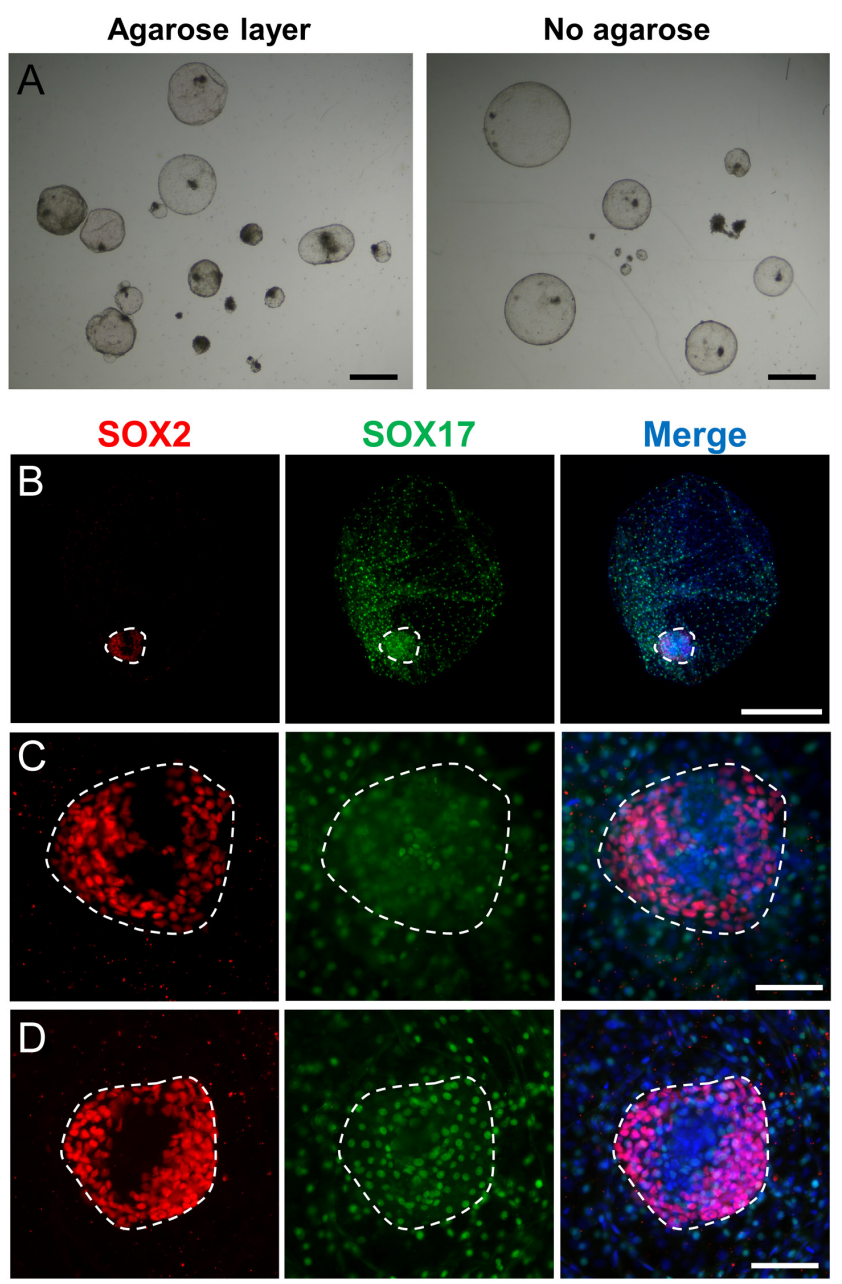

Figure 5 Epiblast development in N2B27 medium. (A) Representative brightfield stereomicroscopic images of D15 embryos developed in N2B27 medium in agarose-coated or agarose-free wells. (B) Epiblast and hypoblast development of a D15 representative embryo cultured in N2B27 in agarose-free well. (C) Magnification of the compact structure resembling an embryonic disc in B. (D) Representative embryonic disc-like structure from a D13 embryo cultured in N2B27 in agarose-free well. Immunofluorescence staining for SOX2 and SOX17; nuclei were counterstained with DAPI (merge). Scale bars= $1 \mathrm{~mm}$ for $\mathrm{A}, 500 \mu \mathrm{m}$ for $\mathrm{B} ; 100 \mu \mathrm{m}$ for magnification in C and D. of epiblast markers evidences a degeneration of this lineage during PHD culture. In agreement with our results, other authors have also reported the abnormal appearance of these cell masses compared to in vivo developed embryonic discs (Machado et al. 2013) and abundant signs of degeneration in the form of apoptosis or necrosis evidenced by transmission electron microscopy in the PHD system (Brandao et al. 2004). Conversely, N2B27 medium supported epiblast survival in $\sim 55-60 \%$ of the embryos, and compact masses of SOX2+ epiblast cells resembling embryonic discs were observed in $\sim 18-25 \%$ of the embryos. The compact embryonic disc-like structures are similar in shape and size to an embryonic disc, but a SOX2-negative area was observed at D15 (Figs 4C and 5C). This SOX2-negative area is already present on D13, starting from the centre of the embryonic disc-like structure (Fig. 5D), suggesting that embryo requirements are still not fully fulfilled in vitro. Although further research is required to mimic maternal conditions, to our knowledge, an in vitro system achieving complete hypoblast migration and the formation of embryonic disc-like structures was not available for any ungulate or livestock species.

The requirement of a relatively high concentration of glucose in post-hatching development media $(5 \mathrm{mg} / \mathrm{mL}$ in PHD and $3.6 \mathrm{mg} / \mathrm{mL}$ in N2B27, Supplementary Table $2)$, absent in the pre-hatching embryo culture media $(0$ $\mathrm{mg} / \mathrm{mL}$, Holm et al. 1999), coincides with an increase in glucose concentration in uterine fluid and an upregulation of different glucose transporters in the endometrium occurring during embryo elongation in ruminants (Forde et al. 2009, Gao et al. 2009a,b, Satterfield et al. 2009). High glucose requirement suggests a metabolic swift occurring after blastocyst hatching (Ramos-lbeas et al. 2019). Our gene expression analysis of key rate-limiting enzymes revealed an increase in the expression of the anaerobic glycolysis enzymes GAPDH and $L D H A$ after culture in PHD medium that was concomitant to a decrease of $C S$, the rate-limiting enzyme for the citric acid cycle (Seedorf et al. 1986), only significant when embryos were cultured free-floating. The high glucose requirement of the embryos seems to be fulfilled by both N2B27 and PHD media, since embryos developed in both media exhibited similar transcript abundance of genes encoding metabolic enzymes. The metabolic switch from oxidative phosphorylation (coupled to citric acid cycle) to anaerobic glycolysis, known as the Warburg effect, has been suggested to play a role on embryo development, arguing that, although energy 
Table 4 Average length, area and volume of surviving embryos cultured in N2B27 medium on agarose-coated vs agarose-free wells at days 11 and 15 of development.

\begin{tabular}{lccccccc}
\hline & $\boldsymbol{n}$ & D11 length $(\mathrm{mm})$ & D11 area $\left(\mathrm{mm}^{2}\right)$ & D11 volume $\left(\mathrm{mm}^{3}\right)$ & D15 length $(\mathrm{mm})$ & D15 area $\left(\mathrm{mm}^{2}\right)$ & D15 volume $\left(\mathrm{mm}^{3}\right)$ \\
\hline Agarose-coated & 11 & $0.64 \pm 0.04$ & $0.35 \pm 0.04$ & $0.16 \pm 0.03$ & $1.07 \pm 0.08$ & $0.92 \pm 0.14$ & $0.76 \pm 0.17$ \\
Agarose-free & 16 & $0.60 \pm 0.05$ & $0.32 \pm 0.04$ & $0.14 \pm 0.04$ & $1.33 \pm 0.1$ & $1.52 \pm 0.26$ & $1.57 \pm 0.40$ \\
\hline
\end{tabular}

No significant differences were found ( $P>0.05, t$-test for D15 length and Mann-Whitney Rank Sum Test for area and volume).

production is less efficient by anaerobic glycolysis, this pathway produces metabolites that may be critical for embryo development and it may reduce the production of reactive oxygen species (ROS) (Bermejo-Alvarez et al. 2010a, Krisher \& Prather 2012). In this sense, the increased expression of CS in embryos cultured inside tunnels compared to those free-floating may entail an increased production of ROS (Zorov et al. 2014). In the same line, an increase in G6PD and its positive regulator SIRT2 (Wang et al. 2014, Lamas-Toranzo et al. 2018) was observed in embryos cultured in tunnel compared with those cultured over agarose layer. Glucose-6phosphate dehydrogenase (G6PD) is the rate-limiting enzyme of the pentose phosphate pathway, a pathway producing the reducing agent $\mathrm{NADPH}$, and thereby its upregulation on embryos cultured inside tunnel may respond to a higher demand for reducing ROS.

In conclusion, the three key developmental processes occurring during early embryonic elongation (trophoblast proliferation, complete hypoblast proliferation and migration, and epiblast development into an embryonic disc-like structure) can be recapitulated in an in vitro system based on N2B27 medium and not requiring agarose tunnel or layer. This system will help to understand the role of specific genes on these developmental processes and could serve as a proxy to determine embryo quality following different in vitro production methodologies.

\section{Supplementary materials}

This is linked to the online version of the paper at https://doi. org/10.1530/REP-20-0243.

\section{Declaration of interest}

The authors declare that there is no conflict of interest that could be perceived as prejudicing the impartiality of the research reported.

\section{Funding}

This work has been funded by the projects StG-757886ELONGAN from the European Research Council and AGL2017-84908-R from the Spanish Ministry of Economy and Competitiveness (MINECO) to P B A. P R I was funded by a Talent Attraction Fellowship from Madrid Region Government (2017-T2/BIO5182) and is currently funded by a Ramón y Cajal Contract from MINECO (RYC2018-025666-I). I L T was funded by a FPI fellowship by MINECO (BES-2015-072774). A $M M$ is funded by an Industrial Doctorate Fellowship (IND2017/BIO7748) from Madrid Region Government.

\section{Author contribution statement}

All authors contributed to embryo production. P R I performed post-hatching embryo culture and molecular analyses. P R I and P B A designed experiments and wrote the manuscript.

\section{Acknowledgement}

The authors want to acknowledge the slaughterhouse 'Transformación Ganadera de Leganés SA' for gently providing bovine ovaries to conduct the experiments.

\section{References}

Alexopoulos NI, Vajta G, Maddox-Hyttel P, French AJ \& Trounson AO 2005 Stereomicroscopic and histological examination of bovine embryos following extended in vitro culture. Reproduction, Fertility, and Development 17 799-808. (https://doi.org/10.1071/rd04104)

Ayalon N 1978 A review of embryonic mortality in cattle. Journal of Reproduction and Fertility $\mathbf{5 4}$ 483-493. (https://doi.org/10.1530/ jrf.0.0540483)

Berg DK, van Leeuwen J, Beaumont S, Berg M \& Pfeffer PL 2010 Embryo loss in cattle between days 7 and 16 of pregnancy. Theriogenology 73 250-260. (https://doi.org/10.1016/j.theriogenology.2009.09.005)

Berg DK, Smith CS, Pearton DJ, Wells DN, Broadhurst R, Donnison M \& Pfeffer PL 2011 Trophectoderm lineage determination in cattle. Developmental Cell $20 \quad 244-255 . \quad$ (https://doi.org/10.1016/j. devcel.2011.01.003)

Bermejo-Alvarez P, Lonergan P, Rizos D \& Gutierrez-Adan A 2010a Low oxygen tension during IVM improves bovine oocyte competence and enhances anaerobic glycolysis. Reproductive Biomedicine Online 20 341-349. (https://doi.org/10.1016/j.rbmo.2009.12.006)

Bermejo-Alvarez P, Rizos D, Rath D, Lonergan P \& Gutierrez-Adan A $2010 \mathrm{~b}$ Sex determines the expression level of one third of the actively expressed genes in bovine blastocysts. PNAS 107 3394-3399. (https:// doi.org/10.1073/pnas.0913843107)

Bermejo-Alvarez P, Rizos D, Lonergan P \& Gutierrez-Adan A 2011 Transcriptional sexual dimorphism in elongating bovine embryos: implications for $\mathrm{XCl}$ and sex determination genes. Reproduction 141 801-808. (https://doi.org/10.1530/REP-11-0006)

Bermejo-Alvarez P, Roberts RM \& Rosenfeld CS 2012 Effect of glucose concentration during in vitro culture of mouse embryos on development to blastocyst, success of embryo transfer, and litter sex ratio. Molecular Reproduction and Development 79 329-336. (https://doi.org/10.1002/ mrd.22028)

Brandao DO, Maddox-Hyttel P, Lovendahl P, Rumpf R, Stringfellow D \& Callesen H 2004 Post hatching development: a novel system for extended in vitro culture of bovine embryos. Biology of Reproduction 71 2048-2055. (https://doi.org/10.1095/biolreprod.103.025916)

Canizo JR, Ynsaurralde Rivolta AE, Vazquez Echegaray C, Suvá M, Alberio V, Aller JF, Guberman AS, Salamone DF, Alberio RH \& Alberio R 2019 A dose-dependent response to MEK inhibition determines hypoblast fate in bovine embryos. BMC Developmental Biology 1913. (https://doi.org/10.1186/s12861-019-0193-9) 
Diskin MG \& Morris DG 2008 Embryonic and early foetal losses in cattle and other ruminants. Reproduction in Domestic Animals 43 (Supplement 2) 260-267. (https://doi.org/10.1111/j.1439-0531.2008.01171.x)

Forde N, Carter F, Fair T, Crowe MA, Evans AC, Spencer TE, Bazer FW, McBride R, Boland MP, O'Gaora P et al. 2009 Progesterone-regulated changes in endometrial gene expression contribute to advanced conceptus development in cattle. Biology of Reproduction 81 784-794. (https://doi.org/10.1095/biolreprod.108.074336)

Gao H, Wu G, Spencer TE, Johnson GA \& Bazer FW 2009a Select nutrients in the ovine uterine lumen. II. Glucose transporters in the uterus and peri-implantation conceptuses. Biology of Reproduction 80 94-104. (https://doi.org/10.1095/biolreprod.108.071654)

Gao H, Wu G, Spencer TE, Johnson GA, Li X \& Bazer FW 2009b Select nutrients in the ovine uterine lumen. I. Amino acids, glucose, and ions in uterine lumenal flushings of cyclic and pregnant ewes. Biology of Reproduction $\mathbf{8 0}$ 86-93. (https://doi.org/10.1095/ biolreprod.108.071597)

Granchi C, Bertini S, Macchia M \& Minutolo F 2010 Inhibitors of lactate dehydrogenase isoforms and their therapeutic potentials. Current Medicinal Chemistry 17 672-697. (https://doi. org/10.2174/092986710790416263)

Helmer SD, Hansen PJ, Thatcher WW, Johnson JW \& Bazer FW 1989 Intrauterine infusion of highly enriched bovine trophoblast protein-1 complex exerts an antiluteolytic effect to extend corpus luteum lifespan in cyclic cattle. Journal of Reproduction and Fertility 87 89-101. (https:// doi.org/10.1530/jrf.0.0870089)

Holm P, Booth PJ, Schmidt MH, Greve T \& Callesen H 1999 High bovine blastocyst development in a static in vitro production system using SOFaa medium supplemented with sodium citrate and myo-inositol with or without serum-proteins. Theriogenology 52 683-700. (https:// doi.org/10.1016/S0093-691X(99)00162-4)

Imakawa K, Anthony RV, Kazemi M, Marotti KR, Polites HG \& Roberts RM 1987 Interferon-like sequence of ovine trophoblast protein secreted by embryonic trophectoderm. Nature 330 377-379. (https://doi. org/10.1038/330377a0)

Khan DR, Dubé D, Gall L, Peynot N, Ruffini S, Laffont L, Le Bourhis D, Degrelle S, Jouneau A \& Duranthon V 2012 Expression of pluripotency master regulators during two key developmental transitions: EGA and early lineage specification in the bovine embryo. PLOS ONE 7 e34110. (https://doi.org/10.1371/journal.pone.0034110)

Krisher RL \& Prather RS 2012 A role for the Warburg effect in preimplantation embryo development: metabolic modification to support rapid cell proliferation. Molecular Reproduction and Development 79 311-320. (https://doi.org/10.1002/mrd.22037)

Lamas-Toranzo I, Pericuesta E \& Bermejo-Alvarez P 2018 Mitochondrial and metabolic adjustments during the final phase of follicular development prior to IVM of bovine oocytes. Theriogenology 119 156-162. (https://doi.org/10.1016/j.theriogenology.2018.07.007)

Machado GM, Ferreira AR, Pivato I, Fidelis A, Spricigo JF, Paulini F, Lucci CM, Franco MM \& Dode MA 2013 Post-hatching development of in vitro bovine embryos from day 7 to 14 in vivo versus in vitro. Molecular Reproduction and Development 80 936-947. (https://doi. org/10.1002/mrd.22230)

Maddox-Hyttel P, Alexopoulos NI, Vajta G, Lewis I, Rogers P, Cann L, Callesen H, Tveden-Nyborg P \& Trounson A 2003 Immunohistochemical and ultrastructural characterization of the initial post-hatching development of bovine embryos. Reproduction 125 607-623. (https:// doi.org/10.1530/rep.0.1250607)

Negrón-Pérez VM, Zhang Y \& Hansen PJ 2017 Single-cell gene expression of the bovine blastocyst. Reproduction 154 627-644. (https://doi. org/10.1530/REP-17-0345)

O'Hara L, Forde N, Kelly AK \& Lonergan P 2014 Effect of bovine blastocyst size at embryo transfer on day 7 on conceptus length on day 14: can supplementary progesterone rescue small embryos? Theriogenology $\mathbf{8 1}$ 1123-1128. (https://doi.org/10.1016/j.theriogenology.2014.01.041)

Ramos-Ibeas P, Calle A, Pericuesta E, Laguna-Barraza R, Moros-Mora R, Lopera-Vasquez R, Maillo V, Yanez-Mo M, Gutierrez-Adan A, Rizos D et al. 2014 An efficient system to establish biopsy-derived trophoblastic cell lines from bovine embryos. Biology of Reproduction 91 15. (https:// doi.org/10.1095/biolreprod.114.118430)

Ramos-Ibeas P, Nichols J \& Alberio R 2018 States and origins of mammalian embryonic pluripotency in vivo and in a dish. Current Topics in Developmental Biology 128 151-179. (https://doi.org/10.1016/ bs.ctdb.2017.11.002)

Ramos-Ibeas P, Sang F, Zhu Q, Tang WWC, Withey S, Klisch D, Wood L, Loose M, Surani MA \& Alberio R 2019 Pluripotency and X chromosome dynamics revealed in pig pre-gastrulating embryos by single cell analysis. Nature Communications 10 500. (https://doi.org/10.1038/ s41467-019-08387-8)

Santos JE, Thatcher WW, Chebel RC, Cerri RL \& Galvao KN 2004 The effect of embryonic death rates in cattle on the efficacy of estrus synchronization programs. Animal Reproduction Science 82-83 513-535. (https://doi.org/10.1016/j.anireprosci.2004.04.015)

Satterfield MC, Song G, Kochan KJ, Riggs PK, Simmons RM, Elsik CG, Adelson DL, Bazer FW, Zhou H \& Spencer TE 2009 Discovery of candidate genes and pathways in the endometrium regulating ovine blastocyst growth and conceptus elongation. Physiological Genomics 39 85-99. (https://doi.org/10.1152/physiolgenomics.00001.2009)

Schindelin J, Arganda-Carreras I, Frise E, Kaynig V, Longair M, Pietzsch T, Preibisch S, Rueden C, Saalfeld S, Schmid B et al. 2012 Fiji: an opensource platform for biological-image analysis. Nature Methods $\mathbf{9}$ 676-682. (https://doi.org/10.1038/nmeth.2019)

Schmittgen TD \& Livak KJ 2008 Analyzing real-time PCR data by the comparative C(T) method. Nature Protocols 3 1101-1108. (https://doi. org/10.1038/nprot.2008.73)

Seedorf U, Leberer E, Kirschbaum BJ \& Pette D 1986 Neural control of gene expression in skeletal muscle. Effects of chronic stimulation on lactate dehydrogenase isoenzymes and citrate synthase. Biochemical Journal 239 115-120. (https://doi.org/10.1042/bj2390115)

Talbot NC, Caperna TJ, Edwards JL, Garrett W, Wells KD \& Ealy AD 2000 Bovine blastocyst-derived trophectoderm and endoderm cell cultures: interferon tau and transferrin expression as respective in vitro markers. Biology of Reproduction 62 235-247. (https://doi.org/10.1095/ biolreprod62.2.235)

Vajta G, Alexopoulos NI \& Callesen H 2004 Rapid growth and elongation of bovine blastocysts in vitro in a three-dimensional gel system. Theriogenology 62 1253-1263. (https://doi.org/10.1016/j. theriogenology.2004.01.007)

van Leeuwen J, Berg DK \& Pfeffer PL 2015 Morphological and gene expression changes in cattle embryos from hatched blastocyst to early gastrulation stages after transfer of in vitro produced embryos. PLOS ONE 10 e0129787. (https://doi.org/10.1371/journal. pone.0129787)

Wang YP, Zhou LS, Zhao YZ, Wang SW, Chen LL, Liu LX, Ling ZQ, Hu FJ, Sun YP, Zhang JY et al. 2014 Regulation of G6PD acetylation by SIRT2 and KAT9 modulates NADPH homeostasis and cell survival during oxidative stress. EMBO Journal 33 1304-1320. (https://doi.org/10.1002/ embj.201387224)

Ying QL \& Smith AG 2003 Defined conditions for neural commitment and differentiation. Methods in Enzymology 365 327-341. (https://doi. org/10.1016/s0076-6879(03)65023-8)

Zorov DB, Juhaszova M \& Sollott SJ 2014 Mitochondrial reactive oxygen species (ROS) and ROS-induced ROS release. Physiological Reviews 94 909-950. (https://doi.org/10.1152/physrev.00026.2013)

Received 1 May 2020

First decision 26 May 2020

Revised manuscript received 18 June 2020

Accepted 17 July 2020 